DOI 10. 18307/2017. 0612

(c) 2017 by Journal of Lake Sciences

\title{
太湖流域江苏片区底栖大型无脊椎动物群落结构及物种多样性”
}

\author{
陈 桥 ${ }^{1}$, 张 翔 ${ }^{1}$, 沈丽娟 ${ }^{1}$, 张小琼 ${ }^{1}$, 周 俊 ${ }^{1}$, 张 咏 $^{2}$, 牛志春 ${ }^{2}$, 徐东昫 ${ }^{1}$ \\ (1: 常州市环境监测中心江苏省环境保护水环境生物监测重点实验室,常州 213001) \\ (2: 江苏省环境监测中心, 南京 210036)
}

\begin{abstract}
摘 要: 为探明现阶段太湖流域江苏片区底栖大型无脊椎动物群落结构及物种多样性, 于 2013 年 $1-3,7-8$ 和 $10-11$ 月对 120 个样点开展了调查. 共记录 6 门 124 科 280 种, 各点位物种数 (4 51 种)、个体密度 (5.5 23363.4 ind. $/ \mathrm{m}^{2}$ ) 和生 物量 $\left(0.1 \sim 6269.2 \mathrm{~g} / \mathrm{m}^{2}\right)$ 差异较大. 不同水体类型的群落结构存在显著差异, 其中溪流以蜉蝣目和毛翅目等水生昆虫为优 势, 水库以摇蚊为优势, 河流以葟毛纲为绝对优势, 湖荡以塞毛纲、摇蚊幼虫和软体动物为优势. 聚类结果显示, 同水体类 型点位的群落结构也呈现不同程度的空间差异. 结合丰度/生物量曲线、特征种及多样性分析, 各聚类组受胁迫程度从轻 到重依次为溪流 (组IX), 太湖敞水区 (组 II ) 和东部水草区 (组 III), 太湖下游湖荡 (组IV)、河流 (组 VI) 和水库 (组 VII), 太 湖上游湖荡 (组 I ) 、其他河流 (组 V) 和水库 (组VIII) 点位. 底栖大型无脊椎动物的分布与物理生境和水质条件呈较好的 空间一致性,生境的多样性和良好的水质条件是保护和恢复物种多样性的关键因素.
\end{abstract}

关键词: 底栖大型无脊椎动物; 群落结构;多样性;胁迫;空间差异;江苏省; 太湖流域

\section{Community structure and species diversity of benthic macroinvertebrates in Taihu Basin of Jiangsu Province}

CHEN Qiao ${ }^{1}$, ZHANG Xiang ${ }^{1}$, SHEN Lijuan ${ }^{1}$, ZHANG Xiaoqiong ${ }^{1}$, ZHOU Jun ${ }^{1}$, ZHANG Yong ${ }^{2}$, NIU Zhichun $^{2} \&$ XU Dongjiong ${ }^{1}$

(1: Jiangsu Environmental Protection Key Laboratory of Aquatic Biomonitoring, Changzhou Environmental Monitoring Center, Changzhou 213001, P.R.China)

(2: Jiangsu Environmental Monitoring Center, Nanjing 210036, P.R.China)

Abstract: To explore the characteristic of community structure and species diversity of benthic macroinvertebrates, samples were collected three times at 120 sampling sites in Taihu Basin of Jiangsu Province. Twenty-nine sampling sites were located in Lake Taihu, and the number of sites in other lakes, rivers, reservoirs and streams were 20, 40, 15 and 16, respectively. The three investigations were conducted in the periods from January to March, from July to August and from October to November in 2013. In total, 280 taxa of benthic macroinvertebrates from 124 families and 6 phyla were recorded. The taxa number (4-51), density ( 5.5-23363.4 ind. $\left./ \mathrm{m}^{2}\right)$ and biomass $\left(0.1-6269.2 \mathrm{~g} / \mathrm{m}^{2}\right)$ varied greatly among sites, and community structure differed significantly among water bodies. In the western hilly region, the community of streams were dominated by aquatic insects from Ephemeroptera, Trichoptera and Odonata, while reservoirs were mainly dominated by Chironomidae larvae. In the eastern plain area, pollution-tolerant oligochaetes dominated the community of rivers, while lakes were dominated by Chironomidae larvae, Oligochaeta and Mollusca. Cluster analysis and One-way analysis of similarity classified the 120 sites into nine groups characterized by different characteristics species, showing the hierarchical gradient of sampling sites in lakes, rivers, reservoirs and streams. Streams(group IX ) were the least stressed by integrating the results of abundance-biomass curves, characteristics species and diversity of each affinity group. Species richness and evenness were relatively lower in central region( group II ) and macrophytes dominated areas ( group III ) of Lake Taihu, whose biomass curves were above the abundance curves and dominated by Mollusca, Malacostraca and Oligochaeta, indicating that the communities suffered relatively weak disturbance. The biomass curves of groups including reservoirs

* 国家水体污染控制与治理科技重大专项 (2012ZX07506-003)、江苏省环保科研基金项目 (2014039, 2014004) 和江 苏省环境监测科研基金项目 (1313,1405) 联合资助. 2017-01-03 收稿; 2017-03-20 收修改稿. 陈桥 (1984 ), 男,硕士,工程师;E-mail: chenqiao_czem@163.com. 
( group VII), lakes ( group IV) and rivers (group VI), which were in the out-flow direction of Lake Taihu, were very close to or intersected with the abundance curves and dominated by Chironomidae larvae, Oligochaeta and Gastropoda, indicating that the communities were moderately disturbed. However, the other groups (group I, V and VIII) were only dominated by Chironomidae larvae and Oligochaeta, indicating intense disturbance. Community structure and spatial distribution of benthic macroinvertebrates in Taihu Basin of Jiangsu Province were strongly correlated to two ecological factors-habitat complexity and water quality, which could be used by managers and policy makers to evaluate and improve restoration practices.

Keywords: Benthic macroinvertebrates; community structure; species diversity; spatial variance; stressor; Jiangsu Province; Taihu Basin

底栖大型无脊椎动物 (下文简称“底栖动物”) 是水生食物链的重要环节, 具有生命周期长、区域性强、 迁移能力弱等特点, 容易受各种环境条件的影响, 被称为 “水下哨兵”, 在生态系统物质循环和能量流动中具 有重要作用 ${ }^{[1]}$. 由于各类群对环境条件变化及污染胁迫响应的差异性,使其占有不同的生态位,因此可以通 过群落结构、优势种类、数量特征等参数来反映环境质量状况 ${ }^{[2]}$. 国内外大型底栖动物方面的研究颇多,包 括群落多样性 ${ }^{[3-10]}$ 、水质评价 ${ }^{[2,11-13]}$ 、水生态健康评估 ${ }^{[1,14-23]}$ 、环境毒理学 ${ }^{[24-25]}$ 以及基准和标准 ${ }^{[26-28]}$ 研究等, 欧美等发达国家已经制定了相关标准或规范 ${ }^{[29-31]}$,为环境管理提供支撑.

太湖流域江苏片区总面积约 $1.94 \times 10^{4} \mathrm{~km}^{2}$, 占全流域的 $52.6 \%$, 是我国城市化和工业化发展速度最快的 区域之一,水污染问题突出,已成为制约社会经济发展的重要因素. 随着国家和省 “水十条”颁布实施, 区域 水环境管理将逐步从水质目标向水生态目标转变,对水生生物指标体系提出了现实且迫切的需求. 实践证 明, 底栖动物相关指标在欧美水环境管理体系中得到了成功应用, 在国内也有较好的基础和积累, 是流域水 生态目标管理体系中的潜在指标之一. 但是, 现有研究主要是针对流域内河流和大型湖泊 ${ }^{[2,9-10,12,32-33]}$, 对流 域内各类型水体还缺乏系统全面的研究. 本文系统研究了太湖流域江苏片区低山丘陵和平原水网区湖荡、 河流、水库及溪流的底栖动物群落结构及多样性,为流域水生态指标体系研究提供系统全面的基础支撑.

\section{1 材料与方法}

\section{1 采样点布设及样品采集与分析}

以流域水质监测网为基础, 兼顾空间全覆盖和代表性等因素, 在太湖流域江苏片区布设 120 个点位 (图 1), 其中太湖 29 个、其他湖荡 20 个、河流 40 个、水库 15 个、溪流 16 个,分别于 2013 年 $1-3 、 7-8$ 和 10-11 月开展监测. 对于湖荡、河流、水库, 样品的采集首先使用 $1 / 16 \mathrm{~m}^{2}$ Peterson 采泥器采集 4 夹, 然后用定长绳索 及竹笔标记一定的距离, 使用三角拖网 (开口边长 $30 \mathrm{~cm}$, 网孔 40 目) 拖曳采集. 拖曳距离视底质淤泥量而 定, 淤泥多时一般 3 5 m, 淤泥少时一般 10 30 m. 现场将采泥器和三角拖网采得的样品分别经 40 目篮网 淘洗后带回实验室进行标本挑拣. 对于溪流, 急流区样品使用踢网 ( $1 \mathrm{~m} \times 1 \mathrm{~m}, 40$ 目) 采集 $1 \mathrm{~m}^{2}$, 并在上下游 各 $50 \mathrm{~m}$ 范围内使用 D 网 (宽 $0.3 \mathrm{~m}, 40$ 目) 采集各类型小生境(如缓流区和静水区等)共计约 $1 \mathrm{~m}^{2}$. 踢网和 D 网采集的标本在现场合并挑拣. 标本用 $4 \%$ 甲醛溶液保存. 参考相关资料 ${ }^{[34-38]}$, 将标本鉴定或区分到种并计 数, 然后用滤纸吸取表面液体, 置于电子天平 (精确至 $0.0001 \mathrm{~g}$ ) 称重, 计算密度和生物量.

\section{2 数据分析}

湖荡、河流和水库的结果综合两种采样方法,其中小个体类群 (如寡毛类、摇蚊幼虫、线虫、等足目和端 足目等) 以采泥器结果计, 较大个体类群 (如软体动物、水生昆虫、十足目等) 以三角拖网结果计. 本研究重点 关注底栖动物的空间格局, 因此将 3 次调查获得的物种名录合并, 个体密度和生物量取算术平均值.

用相对重要性指数 (index of relative importance, IRI) ${ }^{[39]}$ 确定优势种. 采用 ArcGis 10.1 软件绘制空间分 布图. 为了消除量纲差异及偶见种的影响, 使用 PRIMER 5.0 软件将各点位的个体密度进行对数转换, 构建 Bray-Curtis 相似系数矩阵, 采用类平均法 (group average) 对采样点进行聚类分析. 基于聚类结果, 使用 Oneway ANOSIM (analysis of similarities) 检验各聚类组群落结构的差异, 并通过 SIMPER ( similarity percentages) 分析确定对组内相似性贡献较大的种类. 使用丰度/生物量曲线 (abundance-biomass curve, $\mathrm{ABC}$ ) 评估各组底 栖动物群落受到的干扰情况. 同时, 结合 Shannon-Wiener ${ }^{[40]}$ 和 Pielou ${ }^{[4]]} 2$ 个指数分析群落多样性. 


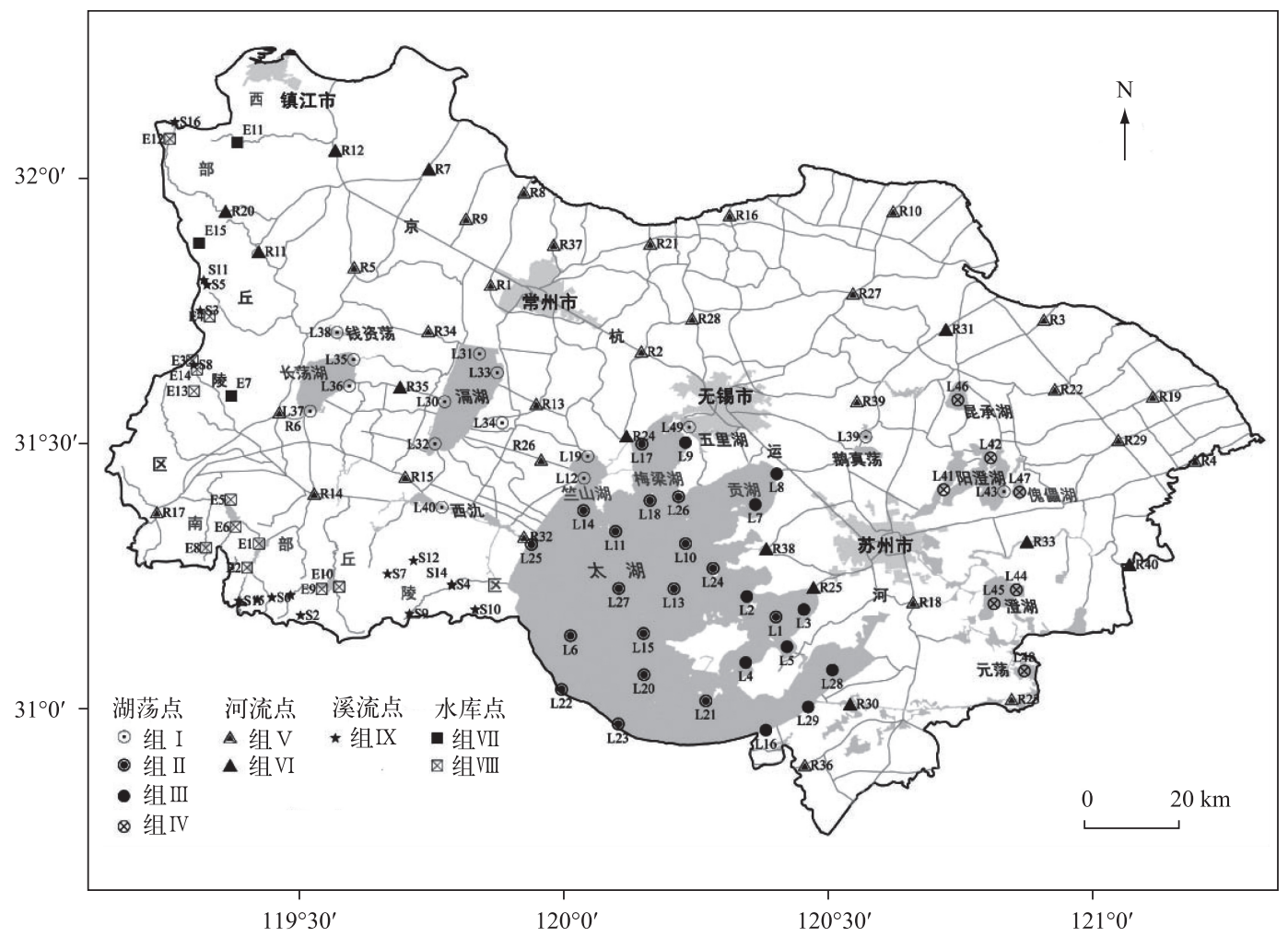

图 1 底栖动物采样点及聚类结果分布

Fig.1 Location of the benthic macroinvertebrates sampling sites and spatial distribution of affinity groups

\section{2 结果}

\section{1 物种组成}

调查共发现底栖动物 280 种, 其中寡毛纲 5 科 24 种, 昆虫纲摇蚊幼虫 51 种、EPTO (蜉蝣目 Ephemeroptera、襀翅目 Plecoptera、毛翅目 Trichoptera 和蜻蜓目 Odonata) 43 科 68 种、其他水生昆虫 31 科 40 种, 软甲纲 13 科 18 种,腹足纲 7 科 29 种,双壳纲 5 科 22 种,多毛纲 5 科 9 种,其他类群 19 种. 不同类型水体的群落结 构存在显著差异 (表 1 和图 2), 其中溪流点位平均物种数最高 (38 种), 平均个体密度最低 ( $305.6 \mathrm{ind} . / \mathrm{m}^{2}$, 昆虫纲占 $78.0 \%$ ), 优势种为纹石蛾科一种、直突摇蚊属一种和梨形环棱螺等; 河流点位平均物种数最低( 17 种), 平均个体密度最高 ( 2642.8 ind. $/ \mathrm{m}^{2}$, 寡毛纲占 $94.4 \%$ ), 霍甫水丝蚓为绝对优势种; 湖荡和水库点位平均 物种数 (23 和 28 种) 和平均个体密度 ( $404.8 \mathrm{ind} . / \mathrm{m}^{2}$, 寡毛纲和摇蚊幼虫占 $73.0 \% ; 389.4 \mathrm{ind} . / \mathrm{m}^{2}$, 摇蚊幼虫 占 $83.5 \%$ ) 居中, 湖荡优势种为霍甫水丝蚓、梨形环棱螺和河蚬等, 水库优势种为长跗摇蚊属一种和齿斑摇 蚊属一种等.

\section{2 空间分布}

各点位底栖动物物种数空间差异较大(图 3A), 流域上游的西部、南部丘陵区和湖泊( 长荡湖、滆湖、太 湖) 下游区域部分点位的物种数较高 ( $26 \sim 51$ 种), 并于宜兴汱溪润 S9 达到最大值 (51 种), 而京杭运河及其 以北的通江河流分类单元数较少 ( 4 24 种), 最小值出现在张家港二干河大桥 R10( 4 种). 个体密度和生物 量的分布也存在较大的空间差异, 且与物种数呈一定的空间对应关系 (图 3B、C), 其中物种数较高的区域总 体表现为较低的个体密度 (平均为 $401.2 \mathrm{ind} . / \mathrm{m}^{2}$ ) 和较高的生物量 (平均为 $251.8 \mathrm{~g} / \mathrm{m}^{2}$ ), 物种数较低的区域 总体表现为较高的个体密度 (平均为 $3526.4 \mathrm{ind} . / \mathrm{m}^{2}$ ) 和较低的生物量 (平均为 $18.8 \mathrm{~g} / \mathrm{m}^{2}$ ). 空间分布格局表 
表 1 太湖流域江苏片区不同类型水体优势种及其相对重要指数

Tab.1 Dominant taxa and their IRI of different types of water bodies in Taihu Basin of Jiangsu Province

\begin{tabular}{|c|c|c|c|c|c|}
\hline \multirow{2}{*}{ 纲 } & \multirow{2}{*}{ 物种* } & \multicolumn{4}{|c|}{$I R I \times 10^{4}$} \\
\hline & & 湖荡 & 河流 & 水库 & 溪流 \\
\hline \multirow[t]{5}{*}{ 寡毛纲 Oligochaeta } & 苏氏尾鳃蚓 Branchiura sowerbyi & & 174 & & \\
\hline & 巨毛水丝蚓 Limnodrilus grandisetosus & & 261 & & \\
\hline & 霍甫水丝蚓 L. hoffmeisteri & 2665 & 6316 & & \\
\hline & 克拉泊水丝蚓 L. claparedeianus & & 405 & & \\
\hline & 颤蚓属一种 Tubifex sp. & & 364 & & \\
\hline \multirow[t]{13}{*}{ 昆虫纲 Insecta } & 菱跗摇蚊属一种 Clinotanypus sp. & & & & 227 \\
\hline & 中国长足摇蚊 Tanypus chinensis & 386 & & 421 & \\
\hline & 前突摇蚊属一种 Procladius sp. & & & 575 & \\
\hline & 长跗摇蚊属一种 Tanytarsus sp. & & & 1243 & \\
\hline & 雕翅摇蚊属一种 Glyptotendipes sp. & & & 412 & \\
\hline & 梯形多足摇蚊 Polypedilum scalaenum & & & 484 & \\
\hline & 小摇蚊属一种 Microchironomus sp. & 397 & & 537 & \\
\hline & 齿斑摇蚊属一种 Stictochironomus sp. & & & 514 & \\
\hline & 恩菲摇蚊属一种 Einfeldia sp. & & & 384 & \\
\hline & 红裸须摇蚊 Propsilocerus akamusi & 462 & & & \\
\hline & 直突摇蚊属一种 Orthocladius sp. & & & & 958 \\
\hline & 似动蜉属一种 Cinygmina sp. & & & & 518 \\
\hline & 纹石蛾科一种 Hydropsychidae sp. & & & & 1821 \\
\hline \multirow[t]{3}{*}{ 软甲纲 Malacostraca } & 米虾属一种 Caridina sp. & & & & 602 \\
\hline & 钩虾属一种 Gammarus sp. & 289 & & & \\
\hline & 太湖大螯蜚 Grandidierella taihuensis & 250 & & & \\
\hline \multirow[t]{6}{*}{ 腹足纲 Gastropoda } & 椭圆萝卜螺 Radix swinhoei & & & & 491 \\
\hline & 折叠夢卜螺 $R$. plicatula & & & & 346 \\
\hline & 放逸短沟蜷 Semisulcospira libertina & & & & 257 \\
\hline & 长角涵螺 Alocinma longicornis & & 144 & & \\
\hline & 梨形环棱螺 Bellamya purificata & 2338 & 2128 & 1690 & 731 \\
\hline & 铜锈环棱螺 B. aeruginosa & 1977 & 1325 & & \\
\hline \multirow[t]{4}{*}{ 双壳纲 Bivalvia } & 圆顶珠蚌 Union douglasiae & & & 906 & \\
\hline & 背角无齿蚌 Anodonta woodiana & & 404 & & \\
\hline & 河蚬 Corbicula fluminea & 2115 & 194 & & \\
\hline & 拉氏蚬 C. largillierti & 501 & & & \\
\hline 扁形动物 Platyhelminthes & 浴虫纲一种 Tubellaria sp. & & & & 371 \\
\hline
\end{tabular}

* 各类型水体中 $I R I$ 排名前十的物种.

明流域上游丘陵地区和湖泊下游部分点位的物种较丰富, 而京杭运河及其以北的通江河流物种较单一, 以 小型的寡毛纲物种为优势类群.

\section{3 聚类及特征种}

基于底栖动物个体密度对各类型水体的点位分别进行聚类分析 (图 4), 共被分为 9 组,其中湖荡 4 组、 河流 2 组、水库 2 组和溪流 1 组. 每两组间的平均相异性百分比介于 $66.2 \% \sim 96.9 \%$ 之间 $(P<0.01)$, 不同类 型水体表现出了不同程度的群落梯度.

湖荡的 49 个点位在 $30 \%$ 的相似性水平上被分为 4 组 (图 4A). 组 I 包括钱资荡、长荡湖、滆湖、竺山湖、 西氿和五里湖等 15 个点位 (图 1), 特征种均为耐污型物种, 包括霍甫水丝蚓、中国长足摇蚊、线铗长足摇蚊、 小摇蚊属一种、苏氏尾鰓蚓和克拉泊水丝蚓,SIMPER 分析表明这 6 种对组内相似性贡献率达 83.6\%(表 2). 

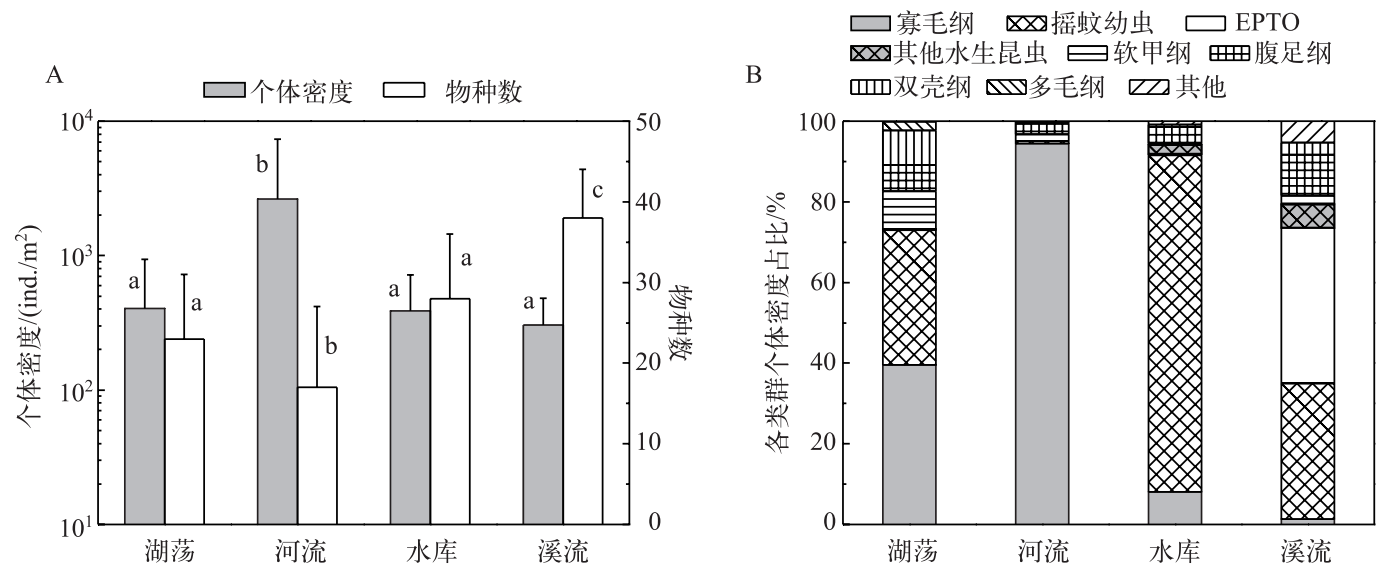

图 2 不同类型水体底栖动物平均物种数、个体密度 $(\mathrm{A})$ 及各类群个体密度占比 $(\mathrm{B})$

(图 A 中不同小写字母表示具有显著差异 (One-way ANOVA, $P<0.05$ ))

Fig.2 Taxa number, density (A) and the proportion of different groups (B) of benthic macroinvertebrates of different types of water bodies

组 II 包括太湖湖心、北部、西部及南部的 17 个点位, 特征种以双壳纲和软甲纲物种为主, 伴有少量塞毛纲和 多毛纲物种,包括河蚬、太湖大鳌蜚、钩虾属一种、杯尾水禹属一种、拉氏蚬、霍甫水丝蚓和背蚓虫, 其对组内 相似性贡献率达 $80.2 \%$. 组正包括太湖东部湖区的 10 个点位,特征种包括梨形环棱螺、铜锈环棱螺、秀丽白 虾、河蚬、钩虾属一种以及寡毛纲、摇蚊幼虫和多毛纲一些种类, 其对组内相似性贡献百分比为 $79.0 \%$. 组 IV 主要包括太湖下游湖群的 7 个点位, 特征种包括红裸须摇蚊、铜锈环棱螺、梨形环棱螺、霍甫水丝蚂、小摇蚊 属一种、前突摇蚊属一种、河蚬, 其对组内相似性贡献百分比为 $81.0 \%$. 河流 40 个点位在 $10 \%$ 的相似性水平 上被分为 2 组 (图 4B), 组 VI主要包括流域上游及长荡湖和太湖等湖泊出湖河流的点位, 共 12 个, 其余归为 组 $V$. 其中, 组 $V$ 的特征种为霍甫水丝蚓和克拉泊水丝蚓, 这 2 种对组内相似性贡献百分比达 $83 \%$; 组 VI的 特征种包括霍甫水丝蚓、梨形环棱螺、颤蚓属一种、苏氏尾鳃蚓等 8 种, 其对组内相似性贡献百分比达 $79.9 \%$. 水库 15 个点位在 $30 \%$ 的相似性水平上被分为 2 组 (图 4C), 组 VII包括 3 个点位, 其余归为组 VIII. 组 VII 的特征种包括颤蚓属一种、梨形环棱螺、长角涵螺、雕翅摇蚊属一种等 9 个物种, 其对组内相似性贡献百分 比为 $72.8 \%$; 组VIII 的特征种以摇蚊为主, 包括小摇蚊属一种、梯形多足摇蚊、前突摇蚊属一种等 9 种摇蚊和霍 甫水丝蚓, 其对组内相似性贡献百分比为 $81.1 \%$. 溪流底栖动物群落未呈现点位间的聚类差异, 因此将溪流 的 16 个点位归为一个组, 即组 IX, 其特征种以水生昆虫为主, 包括蚊石娥科一种、直突摇蚊属一种、似动蜉 属一种、四节蜉科一种、浴虫纲一种等 10 种, 其对组内相似性贡献百分比为 $62.2 \%$. 虽然某一物种 (如霍甫 水丝蚓等) 同时成为多个聚类组的特征种, 但其在不同组的个体密度和相似性贡献百分比存在较大差异,表 明了其在不同生态条件下的生存状态.

\section{4 胁迫程度及多样性}

从各组 $\mathrm{ABC}$ 曲线、多样性和特征种可以看出 (图 5、表 2), 组IX受胁迫相对最轻, 以“蜉蝣目一毛翅目等 水生昆虫” 为优势; 组 II 和 III 胁迫次之, 以“软体一甲壳一寡毛纲” 为优势; 组 IV VI 和 VII胁迫相对较重, 以 “摇蚊幼虫一寡毛纲” 为优势, 伴有少量软体动物; 组 I、V 和VIII胁迫最重, 均为极耐污的小型类群“摇蚊幼 虫一寡毛纲” 为优势. 其中, 组IX虽仍以较清洁的水生昆虫为优势, 但生物量较大的物种缺失, 两个曲线出现 了交叉;代表水库的组 VII 和VIII多样性水平虽高,但主要是因为摇蚊幼虫种类较多.

\section{3 讨论}

\section{1 物理生境对底栖动物空间分布的影响}

底栖动物的空间分布与物理生境条件密切相关 ${ }^{[3-4,9-10,42-43]}$. 太湖流域水网纵横交错,水体类型多样, 溪 

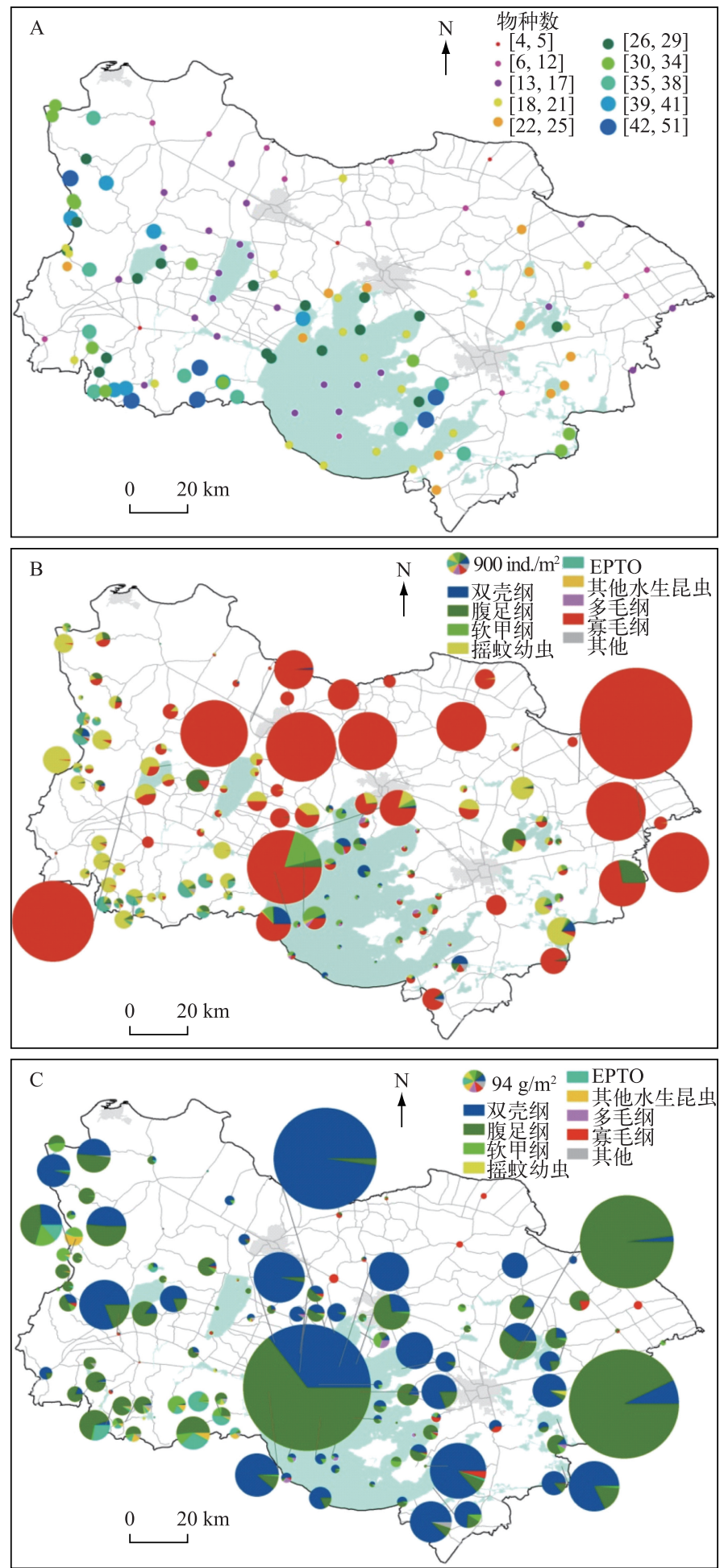

图 3 底栖动物物种数 $(\mathrm{A})$ 、个体密度 $(\mathrm{B})$ 和生物量 $(\mathrm{C})$ 的空间分布

Fig.3 Spatial distribution of taxa number(A), density (B) and biomass (C) of benthic macroinvertebrates 

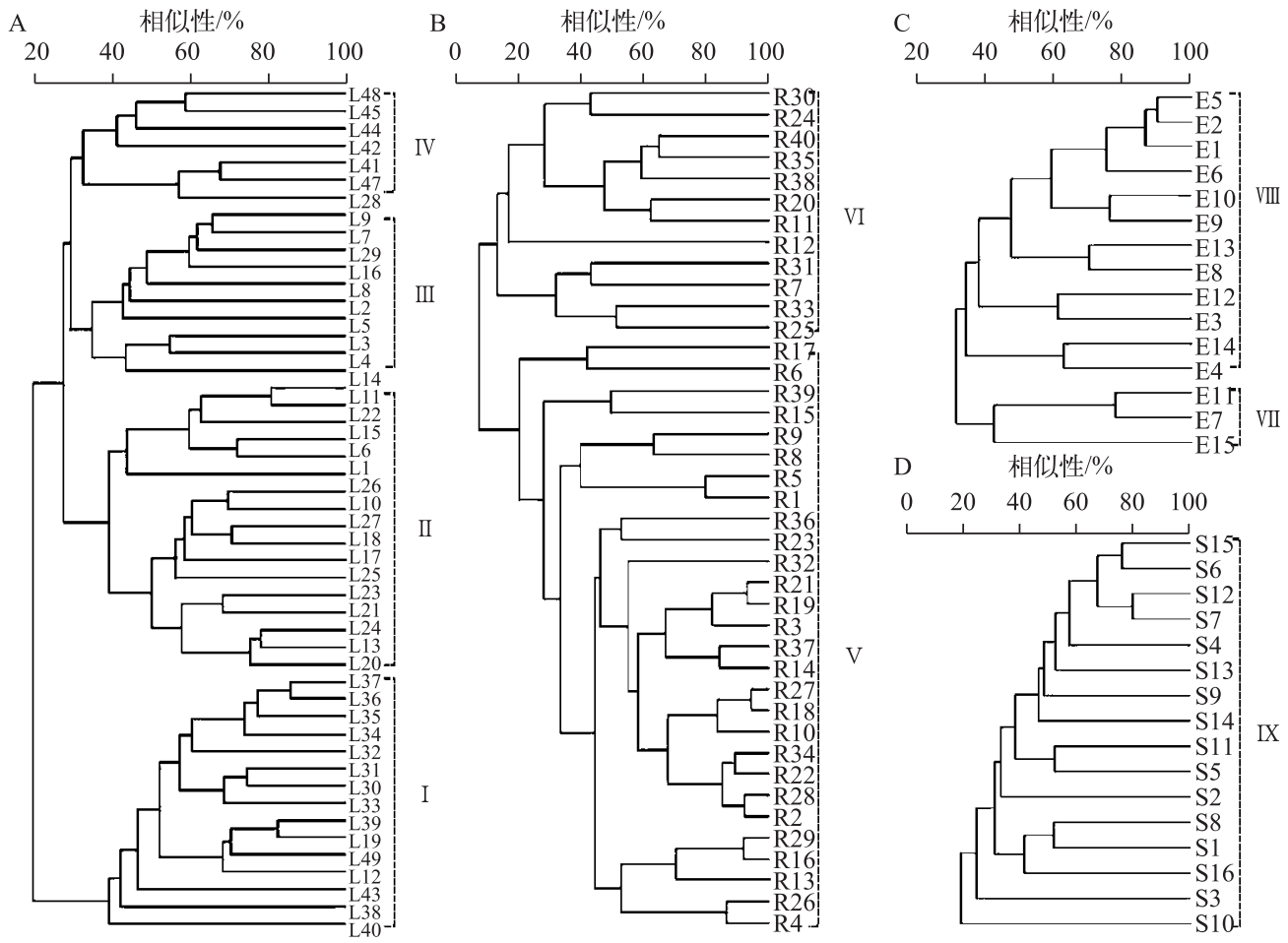

图 4 基于不同类型水体底栖动物密度的 Bray-Curtis 聚类分析树状图 ( $\mathrm{A}$ 湖荡、 $\mathrm{B}$ 河流、 $\mathrm{C}$ 水库、 $\mathrm{D}$ 溪流)

Fig.4 Dendrograms based on Bray-Curtis similarity coefficients of benthic macroinvertebrates density showing the hierarchical clustering of sampling sites in lakes(A), rivers (B), reservoirs(C) and streams (D)

流、水库、河流和湖荡的物理生境差异较大,底栖动物空间分布差异显著 (图 2). 溪流属流域源头水系,位于 上游丘陵区, 植被覆盖度高, 人为干扰较少, 底质以砾石为主, 水流快、溶解氧浓度高, 栖境多样. 因此, 溪流 的物种相对丰富, 以较敏感的毛翅目、蜉蝣目等水生昆虫为优势类群. 这与张又等 ${ }^{[9]}$ 研究结果总体一致, 太 湖流域西部丘陵区的底栖动物群落较东部平原区更丰富, 多样性更高, 且有清水物种分布. 但由于近年资源 开发利用、水利蓄水等力度增大, 生境受到了一定程度破坏, 物种生存空间受到侵占,最易受到冲击的敏感 类群逐步消亡.

水库主要分布在丘陵地区及其与平原的过渡地带, 受人工调蓄影响,消落带水位变化较大, 缺少有利于维 持物种多样性的稳定环境 ${ }^{[44]}$, 同时由于敞水区较深, 底层溶解氧较低,生境单一, 有利于对环境变化适应能力 较强的类群成为优势, 具体表现为摇蚊幼虫平均个体密度占比达 $83.5 \%$. 虽然在局部的滨岸浅水小生境中有较 敏感的水生昆虫和双壳类分布, 但数量较少. 万成炎等 ${ }^{[45]}$ 于 $1998-1999$ 年对江苏省 48 座水库的底栖动物调查 发现,出现频率大于 $80 \%$ 的物种均为小个体耐污类群, 而细蜉属、淡水壳菜等出现频率不足 $10 \%$.

流域内河流是典型的网状结构, 与城市发展密切相关, 受人工改造的痕迹明显 ${ }^{[46]}$, 表现为驳岸硬化、岸 线被裁弯取直、水生植被稀少、底质多为淤泥、栖境单一, 同时还受航运、疏浚、闸控等影响. 相比于其他类型 水体, 河流的生境条件相对最差, 物种多样性最低, 极耐污的颤蚓科平均个体密度达 $2140.6 \mathrm{ind} . / \mathrm{m}^{2}$, 占比达 $67.5 \%$. 根据聚类分组及特征种分析结果, 流域上游和部分湖泊下游点位群落丰富度相对略好, 这可能与这 些点上游的生境 (丘陵植被、湖泊) 具有拦截污染和净化水质等生态缓冲功能有关. 而京杭运河及其以北的 通江河流受城市建设、工业生产及航运等频繁的人类活动干扰, 对群落产生了明显抑制作用. 吴召仕等 ${ }^{[32]}$ 对太湖流域河流底栖动物研究显示, 㘯毛纲个体密度平均占比达 $94.19 \%$, 但位于太湖下游的黄浦江水系物 种较沿江、南河和洮滆水系丰富. 
表 2 各聚类组底栖动物特征种平均密度及其对组内相似性贡献百分比"

Tab.2 Mean density of characteristics species for each affinity group and its contribution to with-in group similarity

\begin{tabular}{|c|c|c|c|c|c|c|c|c|c|c|}
\hline 纲 & 物种 & 组 I & 组 II & 组 III & 组 $I V$ & 组 $V$ & 组VI & 组VII & 组VIII & 组IX \\
\hline 多毛纲 & 齿吻沙蚕属一种 & & & 11.2 & & & & & & \\
\hline \multirow{3}{*}{ Polychaeta } & Nephthys sp. & & & $(11.4 \%)$ & & & & & & \\
\hline & 背蚓虫 & & 7.8 & & & & & & & \\
\hline & Notomastus latericeus & & $(4.4 \%)$ & & & & & & & \\
\hline 寡毛纲 & 苏氏尾鳃蚓| & 6.2 & & 12.3 & & & 36.9 & 13.4 & & \\
\hline \multirow[t]{11}{*}{ Oligochaeta } & Branchiura sowerbyi & $(5.9 \%)$ & & $(16.8 \%)$ & & & $(9.3 \%)$ & $(5.1 \%)$ & & \\
\hline & 霍甫水丝蚛 & 278.2 & 98.8 & 9.9 & 25.1 & 2279.5 & 225.4 & 34.2 & 12.2 & \\
\hline & Limnodrilus hoffmeisteri & $(31.6 \%)$ & $(6.5 \%)$ & $(15.1 \%)$ & $(7.9 \%)$ & $(71.7 \%)$ & $(23.5 \%)$ & $(6.4 \%)$ & $(6.8 \%)$ & \\
\hline & 巨毛水丝蚛 & & & & & & 4.4 & & & \\
\hline & L. grandisetosus & & & & & & $(3.7 \%)$ & & & \\
\hline & 克拉泊水丝蚓 & 24.7 & & & & 233.6 & & & & \\
\hline & L. claparedeianus & $(5.5 \%)$ & & & & $(11.3 \%)$ & & & & \\
\hline & 颤蚓属一种 & & & 5.9 & & & 19.2 & 11.6 & & \\
\hline & Tubifex sp. & & & $(3.8 \%)$ & & & $(9.8 \%)$ & $(12.9 \%)$ & & \\
\hline & 多毛管水蚓 & & & & & & & 4.9 & & \\
\hline & Aulodrilus pluriseta & & & & & & & $(3.1 \%)$ & & \\
\hline 昆虫纲 & 中国长足摇蚊 & 111.9 & & & & & & & 23.2 & \\
\hline \multirow[t]{27}{*}{ Insecta } & Tanypus chinensis & $(22.0 \%)$ & & & & & & & $(4.9 \%)$ & \\
\hline & 线铗长足摇蚊 & 73.0 & & & & & & & & \\
\hline & T. villipennis & $(10.7 \%)$ & & & & & & & & \\
\hline & 前突摇蚊属一种 & & & & 11.6 & & & & 28.5 & \\
\hline & Procladius sp. & & & & $(5.8 \%)$ & & & & $(10.4 \%)$ & \\
\hline & 纳塔摇蚊属一种 & & & & & & & & & 7.3 \\
\hline & Natasia sp. & & & & & & & & & $(3.8 \%)$ \\
\hline & 长跗摇蚊属一种 & & & & & & & & 90.6 & \\
\hline & Tanytarsus sp. & & & & & & & & $(9.4 \%)$ & \\
\hline & 雕翅摇蚊属一种 & & & & & & & 10.7 & 24.6 & \\
\hline & Glyptotendipes sp. & & & & & & & $(5.5 \%)$ & $(4.4 \%)$ & \\
\hline & 羽摇蚊 & & & & & & & & 6.0 & \\
\hline & Chironomus plumosus & & & & & & & & $(4.5 \%)$ & \\
\hline & 梯形多足摇蚊 & & & 3.0 & & & 7.8 & & 25.0 & 5.5 \\
\hline & Polypedilum scalaenum & & & $(4.2 \%)$ & & & $(7.2 \%)$ & & $(11.5 \%)$ & $(3.0 \%)$ \\
\hline & 小摇蚊属一种 & 38.8 & & & 93.8 & & & & 29.2 & \\
\hline & Microchironomus sp. & $(7.9 \%)$ & & & $(7.7 \%)$ & & & & $(16.4 \%)$ & \\
\hline & 隐摇蚊属一种 & & & & & & & & 16.3 & \\
\hline & Cryptochironomus sp. & & & & & & & & $(5.4 \%)$ & \\
\hline & 恩菲摇蚊属一种 & & & & & & & & 22.9 & \\
\hline & Einfeldia sp. & & & & & & & & $(7.4 \%)$ & \\
\hline & 红裸须摇蚊 & & & & 232.6 & & & & & \\
\hline & Propsilocerus akamusi & & & & $(27.5 \%)$ & & & & & \\
\hline & 直突摇蚊属一种 A & & & & & & & & & 29.0 \\
\hline & Orthocladius sp. A & & & & & & & & & $(13.4 \%)$ \\
\hline & 直突摇蚊属一种 B & & & & & & & & & 13.1 \\
\hline & Orthocladius sp. B & & & & & & & & & $(3.5 \%)$ \\
\hline
\end{tabular}


续表 2

\begin{tabular}{|c|c|c|c|c|c|c|c|c|c|c|}
\hline 纲 & 物种 & 组 I & 组 II & 组 III & 组 IV & 组 $V$ & 组 VI & 组 VII & 组VIII & 组IX \\
\hline & 蠓科一种 & & & & & & & 6.8 & & \\
\hline & Ceratopogonidae sp. & & & & & & & $(5.5 \%)$ & & \\
\hline & 四节蜉科一种 & & & & & & & & & 4.8 \\
\hline & Baetidae sp. & & & & & & & & & $(5.1 \%)$ \\
\hline & 似动蜉属一种 & & & & & & & & & 20.3 \\
\hline & Cinygmina sp. & & & & & & & & & $(6.4 \%)$ \\
\hline & 宽基蜉属一种 & & & & & & & & & 8.9 \\
\hline & Choroterpes sp. & & & & & & & & & $(2.8 \%)$ \\
\hline & 纹石蛾科一种 & & & & & & & & & 37.0 \\
\hline & Hydropsychidae sp. & & & & & & & & & $(13.2 \%)$ \\
\hline & 溪泥甲科一种 & & & & & & & & & 3.2 \\
\hline & Elmidae sp. & & & & & & & & & $(2.7 \%)$ \\
\hline \multirow{8}{*}{$\begin{array}{l}\text { 软甲纲 } \\
\text { Malacostraca }\end{array}$} & 杯尾水禹属一种 & & 13.0 & & & & & & & \\
\hline & Cythura sp. & & $(9.7 \%)$ & & & & & & & \\
\hline & 秀丽白虾 & & & 2.2 & & & & & & \\
\hline & Exopalaemon modestus & & & $(4.5 \%)$ & & & & & & \\
\hline & 钩虾属一种 & & 31.9 & 1.5 & & & & & & \\
\hline & Gammarus sp. & & $(13.4 \%)$ & $(4.0 \%)$ & & & & & & \\
\hline & 太湖大螯蜚 & & 39.6 & & & & & & & \\
\hline & Grandidierella taihuensis & & $(16.1 \%)$ & & & & & & & \\
\hline \multirow{8}{*}{$\begin{array}{l}\text { 腹足纲 } \\
\text { Gastropoda }\end{array}$} & 梨形环棱螺 & & & 3.3 & 58.5 & & 66.0 & 12.7 & & \\
\hline & Bellamya purificata & & & $(8.3 \%)$ & $(13.0 \%)$ & & $(16.0 \%)$ & $(12.1 \%)$ & & \\
\hline & 铜锈环棱螺 & & & 1.4 & 61.3 & & 91.1 & 3.7 & & \\
\hline & B. aeruginosa & & & $(4.5 \%)$ & $(14.7 \%)$ & & $(6.3 \%)$ & $(5.6 \%)$ & & \\
\hline & 长角涵螺 & & & & & & 21.9 & 20.5 & & \\
\hline & Alocinma longicornis & & & & & & $(4.1 \%)$ & $(11.4 \%)$ & & \\
\hline & 纹沼螺 & & & & & & & 9.6 & & \\
\hline & Parafossarulus striatulus & & & & & & & $(5.2 \%)$ & & \\
\hline 双壳纲 & 河蚬 & & 63.3 & 3.4 & 15.1 & & & & & \\
\hline \multirow[t]{3}{*}{ Bivalvia } & Corbicula fluminea & & $(23.3 \%)$ & $(6.4 \%)$ & $(4.4 \%)$ & & & & & \\
\hline & 拉氏蚬 & & 14.7 & & & & & & & \\
\hline & C. largillierti & & $(6.8 \%)$ & & & & & & & \\
\hline 扁形动物 & 浴虫纲一种 & & & & & & & & & 12.5 \\
\hline \multirow[t]{2}{*}{ Platyhelminthes } & Tubellaria sp. & & & & & & & & & $(8.3 \%)$ \\
\hline & 合计 & $\begin{array}{c}532.8 \\
(83.6 \%)\end{array}$ & $\begin{array}{c}269.1 \\
(80.2 \%)\end{array}$ & $\begin{array}{c}54.1 \\
(79.0 \%)\end{array}$ & $\begin{array}{c}498.0 \\
(81.0 \%)\end{array}$ & $\begin{array}{c}2513.1 \\
(83.0 \%)\end{array}$ & $\begin{array}{c}472.7 \\
(79.9 \%)\end{array}$ & $\begin{array}{c}128.1 \\
(72.8 \%)\end{array}$ & $\begin{array}{c}278.5 \\
(81.1 \%)\end{array}$ & $\begin{array}{c}141.6 \\
(62.2 \%)\end{array}$ \\
\hline
\end{tabular}

* 括号外数据为平均密度 $\left(\right.$ ind. $\left./ \mathrm{m}^{2}\right)$, 括号内数据为组内相似性贡献百分比.

流域内湖荡众多, 聚类分析将底栖动物群落划分为 4 个聚类组, 分别为竺山湖及其上游湖荡 (组 I )、太 湖敞水区 (组 II) 、太湖东部水草区 ( 组 III) 和太湖下游湖荡 (组 IV). 本研究调查过程中发现, 湖荡物理生境 的空间异质性相对较高, 主要表现为底质类型、水生植被分布等的差异. 1980s 以来,组 I 湖泊(如长荡湖、滆 湖) 受围网养殖、围湖造田等影响 ${ }^{[12]}$, 湖泊生态系统受到严重破坏, 从清水草型转变为浊水藻型生境, 淤泥 黏性底质蓄积严重且富含有机质, 使得底质呈还原状态, 利于耐污能力强的类群形成竞争优势. 对比历史研 究表明, 滆湖由 1990s 初以大型双壳类、蜻蜓和蜉蝣占优势转变为 21 世纪初以寡毛类和摇蚊为优势 ${ }^{[47-48]}$, 对 长荡湖的研究发现其与滆湖的演变历史相似, 推测底栖动物的退化与水生植被破坏有关 ${ }^{[12]}$. 组 II 和组 III 的 划分及群落特点与蔡永久等 ${ }^{[4,10]}$ 、许浩等 ${ }^{[2]}$ 研究结果总体一致, 略有不同的是本研究将太湖梅梁湾归人了敞 

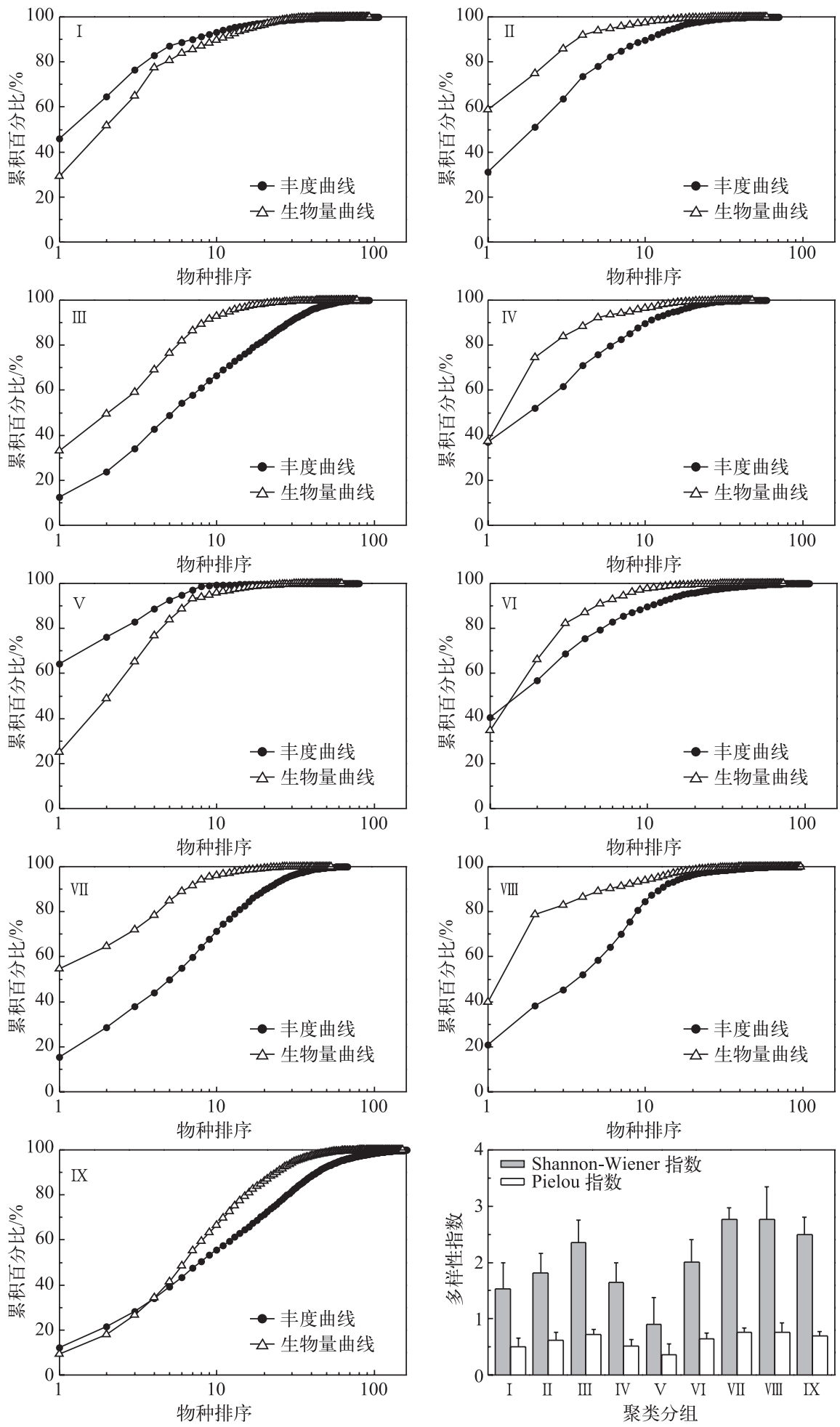

图 5 各聚类组底栖动物群落丰度/生物量曲线及多样性

Fig.5 Abundance-biomass curves and biodiversity indexes of benthic macroinvertebrates for each affinity group 
水区聚类组, 这可能与研究尺度不同有关, 在全流域层面, 梅梁湾底栖动物群落与敞水区相似性更高. 敞水 区扰动大, 底层含氧量较高, 以双壳纲和软甲纲物种为优势, 东部水草区螺类较丰富, 印证了螺-草互利关 系 ${ }^{[49]}$. 组 IV 生境状况介于组 I 和组 III 之间, 受围网养殖等影响, 水生植被呈一定的衰退趋势, 虽有一定量的 螺类分布, 但耐污型的颤蚓和摇蚊幼虫已占据一定优势. 孙月娟 ${ }^{[50]}$ 研究发现阳澄湖底栖动物分布与中华线 鳌蟹的养殖方式相关 ${ }^{[50]}$.

\section{2 水质条件对底栖动物空间分布的影响}

良好的水质条件有利于底栖动物的生存和恢复 ${ }^{[43,51]}$. 据《江苏省环境质量报告书》 ${ }^{[52]}$ 显示, 太湖流域江 苏片区河流水质条件相对较好的点位主要分布在流域上游和湖荡下游, 其中上游河流点位 $(R 11 、 R 20 、 R 7$ 、 $R 12$ 和 R31) 主要受西部丘陵和长江较好来水的影响, 污染相对较轻或基本无明显污染, 湖荡下游点位 $(R 25 、 R 30 、 R 33 、 R 35 、 R 38 、 R 40)$ 可能与湖荡的前置库功能有关 ${ }^{[53-54]}$. 湖泊湿地生态系统具有生态缓冲和自 我净化功能, 是一个天然的 “净化器”, 对上游人湖污染具有拦截净化作用, 出湖水质通常好于人湖水质, 为 下游水体提供了清水通道保障 ${ }^{[55]}$. 因此, 在河流物理生境总体类似的情况下, 位于湖荡下游点位的底栖动 物群落相对较丰富 (图 3A), 与流域上游点共同归到聚类组 VI, 其余河流点位靠近人类活动密集区, 受污染 程度较重,归于聚类组 $V, 2$ 个聚类组群落受干扰状态和特征种存在明显的差异 (图 5 和表 2). 湖荡方面, 由 于太湖环境容量较大, 自我净化能力强, 不仅使太湖各湖区之间水质呈现了显著的差异 ${ }^{[52]}$, 其较好的出水对 下游湖荡水质及水生态状况的保持和改善也起到了积极的作用. 虽然太湖下游湖荡区被开发利用强度也较 大, 城市建设、旅游、围网养殖等不仅对生境造成了破坏, 还增加了污染人湖的风险, 但由于来水较好, 增加 了下游湖荡的环境容量, 提高了生态缓冲能力. 因此, 太湖下游湖荡点位被归为同一聚类组 (组 IV), 且物种 丰富度和多样性均好于太湖上游 (组 I ).

\section{4 结论}

1) 不同类型水体间底栖动物群落结构呈现显著的空间差异. 溪流点位物种丰富度最高, 以较清洁的水 生昆虫为优势类群; 河流相对最低, 以耐污型塞毛纲物种为绝对优势; 湖荡和水库点位物种丰富度居中, 其 中湖荡以软体动物、软甲纲和塞毛纲为优势, 水库以摇蚊幼虫为优势.

2) 同类型水体亦呈现不同程度的空间差异. 聚类分析将湖荡、河流、水库和溪流点位分别划分为 $4 、 2 、 2 、$ 1 个聚类组. 综合各组丰度/生物量曲线、多样性和特征种等, 可以判断出溪流 (组IX) 受胁迫相对最轻, 太湖 敞水区 (组 II) 和东部水草区 (组 III) 次之, 太湖下游湖荡区 (组 IV) 、河流 (组 VI) 和水库 (组 VII ) 胁迫相对较 重,太湖上游湖荡 (组 I ) 、其他河流 (组 V) 和水库 (VIII) 点位胁迫最重.

3) 底栖动物的空间分布格局与物理生境及水质条件相关, 维持生境的多样性和良好的水质条件是保护 和恢复物种多样性的关键.

致谢: 感谢苏州市环境监测中心生态科、南京大学张效伟教授研究团队及青岛正源水生物检测有限公司对 本研究给予的帮助.

\section{5 参考文献}

[ 1 ] Barbour MT, Gerritsen J, Snyder BD et al eds. Rapid bioassessment protocols for use in streams and wadeable rivers: Periphyton, benthic macroinvertebrates and fish, second edition. US Environmental Protection Agency, 1999.

[ 2 ] Xu Hao, Cai Yongjiu, Tang Xiangming et al. Community structure of macrozoobenthos and the evaluation of water environment in Lake Taihu. J Lake Sci, 2015, 27(5) :840-852. DOI:10.18307/2015.0510. [许浩, 蔡永久, 汤祥明等. 太湖 大型底栖动物群落结构与水环境生物评价. 湖泊科学, 2015, 27(5): 840-852.]

[ 3 ] Leung ASL, Dudgeon D. Scales of spatiotemporal variability in macroinvertebrate abundance and diversity in monsoonal streams: detecting environmental change. Freshwater Biology, 2011, 56 (6) : 1193-1208. DOI: 10.1111/j. 1365-2427. 2010.02556.x.

[ 4 ] Cai Y, Gong Z, Qin B. Benthic macroinvertebrate community structure in Lake Taihu, China: Effects of trophic status, wind-induced disturbance and habitat complexity. Journal of Great Lakes Research, 2012, 38(1) : 39-48. DOI: 10.1016/j. jglr.2011.12.009. 
[ 5 ] Zhang Y, Wang B, Han M et al. Relationships between the seasonal variations of macroinvertebrates, and land uses for biomonitoring in the Xitiaoxi River Watershed, China. International Review of Hydrobiology, 2012, 97( 3) : 184-199. DOI: 10.1002/iroh.201111487.

[ 6 ] Qu Xiaodong, Zhang Yuan, Ma Shuqin et al. Spatial distribution characteristics of macroinvertebrate communities in Taizi River Basin. Research of Environmental Sciences, 2013, 26(5) : 509-515. DOI: 10.13198/j.res. 2013.05.48.quxd.001. [渠晓东, 张远, 马淑芹等. 太子河流域大型底栖动物群落结构空间分布特征. 环境科学研究, 2013, 26(5): 509-515.]

[ 7 ] Li Di, Niu Zhichun, Wang Xia et al. Distribution and diversity of macrobenthic communities in the Jiangsu Reach of the Yangtze River. Chinese Journal of Applied and Environmental Biology, 2015, 21(1) : 96-100. DOI:10.3724/SP.J.1145. 2014.06007. [李娣, 牛志春, 王霞等. 长江江苏段底栖动物群落结构与多样性分析. 应用与环境生物学报, 2015, 21( 1 ) : 96-100.]

[8] Zhang Yi, Wang Chouming, Shi Huihua et al. Macrobenthic community succession during last thirty years in Dongting Lake. Ecology and Environmental Sciences, 2015, 24(8): 1348-1353. [张屹, 王丑明, 石慧华等. 洞庭湖近 30 年大型 底栖动物的群落演变. 生态环境学报 2015, 24(8): 1348-1353.]

[ 9 ] Zhang You, Liu Ling, Cai Yongjiu et al. Benthic macroinvertebrate community structure in rivers and streams of Lake Taihu Basin and environmental determinants. China Environmental Science, 2015, 35(5): 1535-1546. [张又, 刘凌, 蔡 永久等. 太湖流域河流及溪流大型底栖动物群落结构及影响因素. 中国环境科学, 2015, 35(5): 1535-1546.]

[10] Cai Yongjiu, Gong Zhijun, Qin Boqiang. Community structure and diversity of macrozoobenthos in Lake Taihu, a large shallow eutrophic lake in China. Biodiversity Science, 2010, 18(1) : 50-59. DOI : 10.3724/SP.J.1003.2010.050. [ 蔡永 久, 龚志军, 秦伯强. 太湖大型底栖动物群落结构及多样性. 生态多样性, 2010. 18(1) : 50-59.]

[11] Liu Lusan, Li Zhongyu, Meng Wei et al. The community structure of zoobenthos and bioassessment of water quality in the lower reaches of the Songhua River. Research of Environmental Sciences, 2007, 20(3) : 81-86. DOI:10.13198/j.res.2007. 03.83.liuls.013. [刘录三, 李中宇, 孟伟等. 松花江下游底栖动物群落结构与水质生物学评价. 环境科学研究, $2007,20(3): 81-86$.

[12] Cai Yongjiu, Liu Jingsong, Dai Xiaoling et al. Community structure of macrozoobenthos and bioassessment of water quality in Lake Changdang, Jiangsu Province. Chinese Journal of Ecology, 2014, 33(5): 1224-1232. [蔡永久, 刘劲松, 戴小 琳等. 长荡湖大型底栖动物群落结构及水质生物学评价. 生态学杂志, 2014, 33(5): 129-138.]

[13] Shu Fengyue, Zhang Chengde, Zhang Chao et al. Community structure of macrozoobenthos and bioassessment of water quality in Lake Nansi. Chinese Journal of Ecology, 2014, 33(1): 184-189. [ 舒凤月, 张承德, 张超等. 南四湖大型底 栖动物群落结构及水质生物学评价. 生态学杂志, 2014, 33(1): 184-189.]

[14] Wang Beixin, Yang Lianfang, Hu Benjin et al. A preliminary study on the assessment of stream ecosystem health in south of Anhui Province using Benthic-Index of Biotic Integrity. Acta Ecologica Sinica, 2005, 25(6): 1481-1490. [王备新, 杨 莲芳, 胡本进等. 应用底栖动物完整性指数 B-IBI 评价溪流健康. 生态学报, 2005, 25(6): 1481-1490.]

[15] Wang Beixin, Yang Lianfang, Liu Zhengwen. Index of biological integrity and its application in health assessment of aquatic ecosystem. Chinese Journal of Ecology, 2006, 25(6) : 707-710. [王备新, 杨莲芳, 刘正文. 生物完整性指数与水生 态系统健康评价. 生态学杂志, 2006. 25(6): 707-710.]

[16] Zhang Yuan, Xu Chengbin, Ma Xiping et al. Biotic integrity index and criteria of benthic organizms in Liao River Basin. Acta Scientiae Circumstantiae, 2007, 27(6):919-927. [张远, 徐成斌, 马溪平等. 辽河流域河流底栖动物完整性评 价指标与标准. 环境科学学报, 2007, 27(6) : 919-927.]

[17] Stoddard JL, Herlihy AT, Peck DV et al. A process for creating multimetric indices for large-scale aquatic surveys. Journal of the North American Benthological Society, 2008, 27(4) : 878-891. DOI: 10.1899/08-053.1.

[18] Ma Taowu, Huang Qinghui, Wang Hai et al. The selection of benthic macroinvertebrate-based multimetrics and preliminary establishment of biocriteria for the bioassessment of the water quality of Taihu Lake. Acta Ecologica Sinica, 2008, 28(3): 1192-1200. [马陶武, 黄清辉, 王海等. 太湖水质评价中底栖动物综合生物指数的笁选及生物基准的确立. 生态学 报, 2008, 28(3): 1192-1200.]

[19] Beck MW, Hatch LK. A review of research on the development of lake indices of biotic integrity. Environmental Reviews, 2009, 17: 21-44. DOI: 10.1139/A09-001.

[20] Weigel BM, Dimick JJ. Development, validation, and application of a macroinvertebrate-based Index of Biotic Integrity for 
nonwadeable rivers of Wisconsin. Journal of the North American Benthological Society, 2011, 30(3) : 665-679. DOI: 10. 1899/10-161.1.

[21] Cai Kun, Zhang Jie, Xu Zhaoan et al. Application of a benthic index of biotic integrity for the ecosystem health assessment of Lake Taihu. J Lake Sci, 2014, 26(1) : 74-82. DOI:10.18307/2014.0109. [ 蔡琨, 张杰, 徐兆安等. 应用底栖动物 完整性指数评价太湖生态健康. 湖泊科学, 2014, 26(1) : 74-82.]

[22] Huang Q, Gao J, Cai Y et al. Development and application of benthic macroinvertebrate-based multimetric indices for the assessment of streams and rivers in the Taihu Basin, China. Ecological Indicators, 2014, 48: 649-659. DOI: 10.1016/j. ecolind.2014.09.014

[23] Moe SJ, Solheim AL, Soszka H et al. Integrated assessment of ecological status and misclassification of lakes: The role of uncertainty and index combination rules. Ecological Indicators, 2015, 48: 605-615. DOI: 10.1016/j.ecolind.2014.08.018.

[24] Shen Jian, Zhao Yin, Li Shaonan et al. Acute toxicity of three common pesticides to Bellamya quadrata, Cipangopaludina cathayensis and Corbicula fluminea. Chinese Journal of Pesticide Science, 2013, 15(5): 559-566. [沈坚, 赵渘, 李少南 等. 三种常用农药对环棱螺、圆田螺和河蚬的急性毒性研究. 农药学学报, 2013, 15(5) : 559-566. ]

[25] Liu Li, Zhong Wenjue, Zhu Lingyan. Chronic effects of Hexachlorobenzen (HCB) in sediments to Chironomus kiiensis Larvae. Asian Journal of Ecotoxicology, 2014, 9(2): 261-267. DOI:10.7524/AJE.1673-5897.20130820001. [刘丽, 钟文 玨, 祝凌燕. 沉积物中六氯苯对摇蚊幼虫的慢性毒性效应. 生态毒理学报, 2014, 9(2) : 261-267. ]

[26] Chen Yanqing, Meng Wei, Wu Xuefang et al. Ambient water quality criteria system in the United States. Research of Environmental Sciences, 2011, 24(4) : 467-474. [ 陈艳卿, 孟伟, 武雪芳等. 美国水环境质量基准体系. 环境科学研究, $2011,24(4): 467-474$.

[27] Liu Zhengtao, Wang Xiaonan, Yan Zhenguang et al. Discussion of minimum "3 Phyla and 6 Families” toxicity data requirements for deriving water quality criteria. Research of Environmental Sciences, 2012, 25(12) : 1364-1369. [刘征涛, 王晓南, 间振广等. “三门六科”水质基准最少毒性数据需求原则. 环境科学研究, 2012, 25(12)：1364-1369.]

[28] Zhang Lingsong, Wang Yeyao, Meng Fansheng et al. Species selection methods in deriving water quality criteria for aquatic life. Environmental Science, 2014, 35(10) : 3959-3969. [张铃松, 王业耀, 孟凡生等. 水生生物基准推导中物种选择 方法研究. 环境科学, 2014, 35(10): 3959-3969.]

[29] International Organization for Standardization. Water quality-Guidelines for the selection of sampling methods and devices for benthic macroinvertebrates in fresh waters, ISO 10870, 2012.

[30 ] International Organization for Standardization. Water quality-Biological classification of rivers- Part 1: Guidance on the interpretation of biological quality data from surveys of benthic macroinvertebrates, ISO 8689-1, 2000.

[31] APHA. Standard Methods for the Examination of Water and Wastewater, 22nd edition. Washington, DC: American Public Health Association, 2012:10-67-10-79.

[32] Wu Zhaoshi, Cai Yongjiu, Chen Yuwei et al. Assemblage structure investigation of macrozoobenthos and water quality bioassessment of the main river systems in Taihu Basin. J Lake Sci, 2011, 23(5) : 686-694. DOI: 10.18307/2011.0504. [ 吴 召仕, 蔡永久, 陈宇炜等. 太湖流域主要河流大型底栖动物群落结构及水质生物学评价. 湖泊科学, 2011, 23 (5): 686-694.]

[33] Gao Xin, Niu Cuijuan, Hu Zhongjun. Macrobenthos community structure and its relations with environmental factors in Taihu basin. Chinese Journal of Applied Ecology, 2011, 22(12): 3329-3336. [高欣, 牛翠娟, 胡忠军. 太湖流域大型 底栖动物群落结构及其与环境因子的关系. 应用生态学报, 2011, 22(12): 3329-3336.]

[34] Liu Yueying, Zhang Wenzhen, Wang Yuexian et al eds. Economic Fauna of China: Freshwater Mollusca. Beijing: Science Press, 1979. [刘月英, 张文珍, 王跃先等. 中国经济动物志: 淡水软体动物. 北京: 科学出版社, 1979.]

[35] He Zhihui, Yan Shengliang, Yang Hequan et al eds. Freshwater biology. I : taxonomy. Beijing: Agriculture Press, 1982: 181-334. [何志辉, 严生良, 杨和荃等. 淡水生物学( 上册分类学部分). 北京: 农业出版社, 1982: 181-334.]

[36] Morse JC, Yang L, Tian L eds. Aquatic insects of China useful for monitoring water quality. Nanjing: Hohai University Press, 1994.

[37] Yang Tong ed. Fauna Sinica: Annelida, Hirudinea. Beijing: Science Press, 1996. [杨潼. 中国动物志: 环节动物门, 蛭纲. 北京: 科学出版社, 1996.]

[38] Wang Hongzhu ed. Studies on taxonomy, distribution and ecology of microdrileoligochaetes of China, with descriptions of two new species from the vicinity of the Great Wall Station of China, Antarctica. Beijing: Higher Education Press, 2002. 
[王洪铸. 中国小蚓类研究:附中国南极长城站附近地区两新种. 北京: 高等教育出版社, 2002.]

[39] Han Jie, Zhang Zhinan, Yu Zishan. Macrobenthic community structure in the southern and central Bohai Sea, China. Acta Ecologica Sinica, 2004, 24(3): 531-537. [ 韩洁, 张志南, 于子山. 渤海中、南部大型底栖动物的群落结构. 生态学 报, 2004, 24(3): 531-537.]

[40] Shannon CE, Weaver W eds. The mathematical theory of communication. Urbana: University of Illinois Press, 1949.

[41] Pielou EC eds. Ecological Diversity. NewYork: Wiley-Inters, 1975.

[42] Wang B, Liu D, Liu S et al. Impacts of urbanization on stream habitats and macroinvertebrate communities in the tributaries of Qiangtang River, China. Hydrobiologia, 2012, 680(1) : 39-51. DOI:10.1007/s10750-011-0899-6.

[43] Cai Y, Zhang Y, Wu Z et al. Composition, diversity, and environmental correlates of benthic macroinvertebrate communities in the five largest freshwater lakes of China. Hydrobiologia, 2017, 788 (1) : 85-98. DOI: 10. 1007/s10750-0162989-y.

[44] Han Boping. Reservoir ecology and limnology in China: A retrospective comment. J Lake Sci, 2010, 22(2) : 151-160. DOI: 10.18307/2010.0201. [ 韩博平. 中国水库生态学研究的回顾与展望. 湖泊科学, 2010, 22(2): 151-160.]

[45] Wang Chengyan, Wu Xiaohui, Hu Chuanlin et al. Investigation and comprehensive assessment of zoobenthos of reservoirs in Jiangsu Province. J Lake Sci, 2004, 16(1): 44-49. DOI:10.18307/2004.0106. [万成炎, 吴晓辉, 胡传林等. 江苏 省水库底栖动物调查及其综合评价. 湖泊科学, $2004,16(1): 44-49$. ]

[46] Liu Hua. Assessment of typical river habitat quality in Taihu Lake Basin [Dissertation]. Nanjing: Nanjing University, 2012. [刘华. 太湖流域典型河流生境质量评价研究 [ 学位论文]. 南京: 南京大学, 2012.]

[47] Liu Qigen, Kong Youjia, Chen Liqiao et al. Effect of pen aquaculture on community structure and species diversity of zoobenthos in Gehu Lake. Chinese Journal of Applied and Environmental Biology, 2005, 11(5) : 566-570. [刘其根, 孔尤佳, 陈立侨等. 围网养殖对滆湖底栖动物群落组成及物种多样性的影响. 应用与环境生物学报, 2005, 11(5): 566-570. ]

[48] Wang Liqing, Wu Liang, Zhang Ruilei. Spatiotemporal variation of zoobenthos community and bio-assessment of water quality in Gehu Lake. Chinese Journal of Ecology, 2012, 31(8) : 1990-1996. [王立卿, 吴亮, 张瑞雷等. 滆湖底栖动 物群落的时空变化及水质生物学评价. 生态学杂志, 2012, 31(8): 1990-1996.]

[49] Li Kuanyi, Wen Mingzhang, Yang Hongwei et al. Mutualistic relationship between freshwater snails and aquatic macrophytes. Acta Ecologica Sinica, 2007, 27 (12) : 5427-5432. [李宽意, 文明章, 杨宏伟等. “螺一草” 的互利关系. 生态 学报, 2007, 27(12): 5427-5432.]

[50] Sun Yuejuan. Community ecology of macrozoobenthos in Mingzhu Lake, Shanghai and Yangcheng Lake, Jiangsu Province [Dissertation]. Shanghai: Shanghai Ocean University, 2011. [孙月娟. 上海明珠湖与江苏阳澄湖大型底栖动物群落 生态学研究 [学位论文]. 上海: 上海海洋大学, 2011.]

[51] Vilmi A, Karjalainen SM, Kuoppala M et al. Taxonomic distinctness along nutrient gradients: More diverse, less diverse or not different from random? Ecological Indicators, 2015, 61: 1033-1041. DOI:10.1016/j.ecolind.2015.10.061.

[52] Environmental Protection Department of Jiangsu Province ed. Jiangsu environmental quality report ( $2011-2015)$. Nanjing: Hohai University Press, 2016: 132-135. [江苏省环境保护厅. 江苏省环境质量报告书 $(2011-2015)$. 南 京: 河海大学出版社, 2016: 132-135.]

[53] Zhang Yongchun, Zhang Yimin, Hu Mengchun et al. Studies on front damming technology for NPS pollution control of river network in plain areas. China Water Resources, 2006, 17: 14-18. [张永春, 张毅敏, 胡孟春等. 平原河网地区面源 污染控制的前置库技术研究. 中国水利, 2006, 17: 14-18.]

[54] Zhang Yimin, Duan Jincheng, Chao Jianying et al. Application of estuary pre-dam to eutrophication control in Gehu Lake. Journal of Ecology and Rural Environment, 2013, 29(3): 273-277. [张毅敏, 段金程, 晁建顷等. 河口前置库系统在 滆湖富营养化控制中的应用研究. 生态与农村环境学报, 2013, 29(3): 273-277.]

[55] Wang Yan, Huang Jiacong, Yan Renhua et al. Nutrient removal efficiency of lake wetlands: A case study of Sanshan Wetland in Lake Taihu, eastern China. J Lake Sci, 2016, 28(1) : 124-131. DOI: 10.18307/2016.0114. [王雁, 黄佳㙂, 用 人华等. 湖泊湿地的水质净化效应——以太湖三山湿地为例. 湖泊科学, 2016, 28(1) : 124-131.] 